ArtefaCToS. Revista de estudios de la ciencia y la tecnología

eISSN: $1989-3612$

Vol. 8, No. 2 (2019), 2. ápoca, 139-160

DOI: http://dx.doi.org/10.14201/art201982139160

\title{
El a priori relativo. ¿Hacia una noción pragmatista de la ciencia?
}

\section{The relative a priori. Towards a pragmatist conception of science?}

\section{Mariano SANJUAN SALINAS}

Universidad Autónoma de Madrid, España

mariano.sanjuan@uam.es

Recibido: 05/07/2019. Revisado: 29/07/2019. Aceptado: 11/10/2019

\section{Resumen}

Se propone una revisión del concepto de a priori relativo desde la concepción de Michael Friedman. Se comenzará exponiendo los aspectos de las obras de Carnap y Kuhn que cimientan las dimensiones estática y dinámica de la obra de Friedman. Se argumentará que, a pesar de las virtudes de su interpretación, la falta de sensibilidad pragmática le impide caracterizar adecuadamente la elección teórica. A continuación, se expondrán varias críticas a la elucidación de Friedman. Por último, a raíz de tales críticas, se explorarán lecturas pragmatistas del a priori relativo como superación de las dificultades analizadas.

Palabras clave: Michael Friedman; a priori constitutivo; pragmatismo; elección teórica; racionalidad comunicativa.

\begin{abstract}
I put forward a revision of the concept of the relative a priori from Michael Friedman's proposal. I will begin by exposing those aspects of Carnap and Kuhn that underlie the static and dynamic dimensions of Friedman's project. It will be argued that, despite its virtues, the lack of pragmatic awareness prevents him from properly characterize theory choice. Hereafter, some criticism will be presented. Lastly, stemming from criticism, some pragmatic readings of the relative a priori will be explored as overcoming the issues addressed.
\end{abstract}

Keywords: Michael Friedman; Constitutive A Priori; Pragmatism; Theory Choice; Communicative Rationality. 


\section{Introducción ${ }^{1}$}

Hace poco más de siglo y medio, la enunciación de geometrías alternativas a partir de la supresión del quinto postulado de Euclides y el posterior desarrollo de la mecánica relativista (en el seno de una de esas geometrías, la de Riemann), pusieron bajo sospecha que al espacio le correspondiese la estructura que Newton y Kant habían supuesto ${ }^{2}$, y máxime, que espacio y tiempo fueran formas puras de la intuición (Kant, 1787, A24/B39 y A33-34/B50). Si era demostrable que el tipo de relación que mantienen espacio y sistemas geométricos no es necesaria, entonces no parecían quedar motivos para confiar en la existencia de conocimiento a priori. De acuerdo con Coffa $(1991,2)$, esta crisis ha permeado transversalmente la epistemología y filosofía de la ciencia del siglo XX. En un extremo, los integrantes del Círculo de Viena se esforzaron en rebatir lo que Schlick llamó en alguna ocasión nebulosas metafísicas, entre las que se encontraba el conocimiento a priori kantiano (Schlick, 1925, \$38-39). En el otro, desde la escuela de Maburgo se trató de salvaguardar las intuiciones trascendentales de la filosofía de Kant para hacerlas compatibles con la nueva ciencia. Así las cosas, a lo largo del pasado siglo y en lo transcurrido del presente no ha habido consenso sobre una respuesta unitaria, pero sí sobre cuál es la pregunta central: ¿existe el conocimiento a priori?

Una respuesta intermedia a los dos polos es la concepción relativa del a priori. Quizá Reichenbach fuera el primero en ofrecer una versión suficientemente completa. En su elucidación, Reichenbach ofrece una interpretación de la obra kantiana en la que el término "a priori" posee dos significados diferentes: "a priori" en tanto "necesariamente verdadero" o "trascendentalmente verdadero" y "a priori" en tanto "constitutivo de la experiencia" (Reichenbach, 1920, 48-49). Con motivo de acomodar los sucesos revolucionarios anteriormente descritos a una teoría del a priori, Reichenbach asume el segundo sentido y rechaza el primero. Una consecuencia directa de este hecho es que los principios a priori no se definen por su carácter necesario y trascendental: “a priori”", afirma Reichen-

\footnotetext{
${ }^{1}$ Una versión anterior del artículo se presentó en las II Jornadas Novatores en la Universidad de Salamanca el 4 de abril de 2019. Agradezco encarecidamente los enriquecedores comentarios de la audiencia, y en particular, las juiciosas aportaciones de Ángel Manuel Faerna, Ángeles J. Perona, María José Frapolli y Ramón del Castillo. También agradezco las observaciones de Paula Olmos a borradores previos del presente trabajo y de dos revisores anónimos de la revista ArtefaCToS.

${ }^{2}$ Kant veía en la física de Newton una explicación acertada del comportamiento de los objetos de la percepción: ambos entendían el espacio en términos de una estructura tridimensional euclideana, defendían el principio de conservación de la masa, la ley de inercia (primera ley de Newton) y la ley de acción-reacción (tercera ley en la mecánica de Newton) (Friedman, 1994). Sin embargo, Newton consideraba que el espacio y el tiempo eran entidades absolutas, confiriéndoles así una identidad ontológica propia. Este es un supuesto que Kant no podía aceptar. De ahí que emprendiese el camino de la justificación metafísica de una ciencia que en los aspectos anteriores sí consideraba cierta, argumentando que tiempo y espacio no son entidades absolutas sino la forma de la intuición del sujeto trascendental.
} 
bach, "significa 'anterior al conocimiento', pero no 'para siempre' ni tampoco 'independiente de la experiencia”" $(1920,105)$. Esto dará lugar en su concepción a que los principios a priori varíen en episodios de revoluciones científicas (y por ello sean revisables) y a que cumplan un rol a priori exclusivamente en la teoría de la que forman parte (y por ello sean relativos). Lo que se produce en la reformulación de Reichenbach es la privación de todo cariz metafísico al concepto de aprioricidad $^{3}$.

Consecuentemente, al disociar el conocimiento a priori de las ideas tradicionales de universalidad, atemporalidad y trascendentalidad, lo que se sitúa en el centro de la reflexión es la idea de constitución. Según Reichenbach (1920, 53-56), la función de constitución se realiza mediante lo que denomina axiomas coordinativos, cuyo cometido es coordinar la aplicación de sistemas de entidades conceptuales (viz. sistemas matemáticos y geométricos) a fenómenos empíricos. Estos axiomas son, en síntesis, principios encargados de dotar de significado empírico a los enunciados de la matemática abstracta; un "tercero mediador", en palabras de Ryckman (2005), entre las teorías físicas y los objetos de la experiencia. Así, la idea de constitución se define como la ordenación de los datos de la percepción en base a ciertos principios de la teoría, como la métrica euclideana o la noción de tiempo absoluto en el caso de la mecánica de Newton, o el principio de equivalencia y la constante de la luz en la mecánica relativista. Estos principios actúan de manera previa a la generación de conocimiento empírico, de manera semejante a cómo las formas puras del entendimiento y de la intuición fijaban las condiciones de posibilidad del conocimiento para Kant. La gran diferencia, debe recordarse, es que para Reichenbach dichos principios no son trascendentales ni apodícticos $^{4}$. Recogiendo el testigo de Reichenbach, Friedman (2001, 73-74) mantiene que la función constitutiva de ciertos principios consiste en "asentar las condiciones necesarias bajo las cuales el conocimiento empírico puede tener

\footnotetext{
${ }^{3}$ Uno podría preguntarse, al hilo de lo expuesto, por la relación que se establece entre lo a priori y lo necesario en el marco del a priori relativo. Aunque no disponga del espacio para tratar tal asunto con detenimiento, será un tema latente y consustancial al artículo que será desvelado progresivamente. De la misma manera que Kripke ha defendido que existen enunciados necesarios a posteriori y enunciados contingentes a priori (1980, 170 y ss., y 34 y ss. respectivamente), los defensores del a priori relativo entienden que la relación entre aprioricidad y necesidad es compleja, pero desde un enfoque completamente diferente. Dada una teoría científica, existen una serie de principios que todo enunciado empírico debe satisfacer. En tanto que éstos son presuposiciones de la teoría, son necesarios. Pero dicha concepción sostiene también que tales principios son susceptibles de ser modificados. Entonces, si definimos lo necesario como lo "universalmente válido" o "válido en todo tiempo y mundo posible", los principios a priori relativos no lo son. A decir verdad, este es un debate que aparece en contadas ocasiones en la literatura sobre el a priori relativo, asumiendo que el quid de la cuestión no es hasta qué punto lo a priori está vinculado a lo necesario, si no qué función cumplen ciertos principios en las teorías científicas y si tal función merece ser calificada de apriorística.
}

${ }^{4}$ Véase De Pierris (1992) para una lectura de la idea de constitución afín a la de Reichenbach. Para una contraparte crítica, véase Ryckman (2005, 28 y ss.) y Padovani (2011). 
lugar", entendiendo por "condiciones necesarias" el marco espacio-temporal de referencia en el que operan las leyes dinámicas (Ryckman, 2005, 245). En esta línea, postula Friedman, "afirmar que A es una condición constitutiva de B" significa que A es una condición necesaria "no solo de la verdad de B, sino de la significatividad de B o de su capacidad de poseer valor de verdad". La teoría más relevante a este respecto (y con más relevante denoto más leída y discutida) ha sido precisamente la de Michael Friedman. En lo que sigue, defenderé dos argumentos principales: que su explicación a la elección de teorías es insatisfactoria y que una concepción de índole pragmatista es preferible.

\section{Michael Friedman y las razones de su dinámica}

Michael Friedman $(2001 ; 2002 ; 2007 ; 2011)$ ha desarrollado teoría sobre el desarrollo racional del conocimiento científico basada en la identificación de sus elementos apriorísticos. A continuación, sintetizaré las líneas esenciales de su propuesta, analizando la influencia de las obras de Carnap $(1934 ; 1950)$ y Kuhn (1962). La exposición me permitirá sostener, apoyándome en Richardson (2010), que Friedman abogó por una concepción racionalista de la ciencia fruto del deseo de evitar una caracterización pragmatista del cambio teórico.

\subsection{Carnap, Friedman, y los marcos lingüísticos}

Friedman siempre se ha manifestado partidario de la idea, popularizada por él mismo (Friedman, 1999, 69-70; 2007, 101 y ss.), de que la obra de Carnap exhibe una significativa huella neokantiana. Según relata Coffa $(1991,207)$, en una carta dirigida a Dingler en 1920, Carnap menciona a Kant, Helmholtz y Natorp, entre otros, como ejes fundamentales de su estudio. Pero si de alguna forma puede sintetizarse el sedimento kantiano en la obra de Carnap es a través de las palabras de Peláez Cedrés (2008, 144; énfasis original): "en la filosofía de Carnap lo formal toma el papel de lo trascendental, no en el sentido de las formas subjetivas universales y necesarias pertenecientes al sujeto trascendental, sino en su papel de convenciones con valor constitutivo". Así las cosas, Friedman ve en la filosofía de Carnap una apuesta por la concepción relativa del a priori, como continuación de la de Reichenbach, y como anticipación de la suya propia. Cabe matizar que sería inapropiado localizar a Carnap bajo la sombra exclusiva del neokantismo, tanto como injusta es la tradicional caricatura del neopositivismo naíf. Carnap renunció deliberadamente a la existencia de juicios sintéticos a priori, pero también receló del empirismo radical, manteniendo la idea de que el conocimiento está constituido en algún sentido del término. Esta intuición está presente desde al menos la publicación del Afbau, pero es particularmente sensible en Carnap (1934) y (1950). 
Uno de sus objetivos primarios fue edificar un sistema lógico para identificar satisfactoriamente las partes "formales" y "descriptivas" del lenguaje científico. Con esto en mente, Carnap $(1934, \$ 51)$ sostuvo que todo sistema lingüístico $S$ contiene dos tipos de postulados o reglas de inferencia que bautizó como reglas de transformación: "las reglas de transformación de $S$ lógico-matemáticas", denominadas reglas-L, y "el resto de reglas, físicas", nombradas como reglas-P. Reglas-L y reglas-P poseen un estatus epistémico bien diferenciado, pues las primeras establecen los estándares de "veracidad" y "validez" de las segundas. De esta manera, Carnap asume que las reglas-L de un sistema $S$ son analíticas, mientras que la "parte descriptiva" formada por las reglas-P, es sintética. La originalidad de su propuesta radica en la tesis que defiende que toda lógica bien formada es igualmente correcta. Esto implica la existencia de un margen de capacidad deliberativa sobre el lenguaje que se pretende utilizar siempre que se cumpla con unos criterios mínimos de claridad metodológica y se proporcionen reglas sintácticas en lugar de argumentos filosóficos (Carnap, 1934, \$17). Así, tan solo se está impelido por las normas sintácticas de un grupo de reglas-L cuando se ha decidido adoptarlas. Esta es la gran novedad que introdujo Carnap frente a otros logicistas anteriores: el hecho de relativizar o convencionalizar el carácter apodíctico de la lógica al sistema en que se aplica 5 . Este fenómeno es lo que Carnap llamó el principio de tolerancia. Más adelante, Carnap (1950) recuperó la preocupación por la relación entre la parte constitutiva de lo que denominó un "marco lingüístico" y la parte constituida:

Si uno desea hablar en su lenguaje sobre un nuevo tipo de entidades, debe introducir un sistema de nuevas formas de hablar, sujeto a nuevas reglas; podemos llamar a este procedimiento la construcción de un marco lingüístico para las entidades en cuestión. $\mathrm{Y}$ ahora debemos distinguir dos tipos de preguntas sobre existencia: primero, preguntas sobre la existencia de ciertas entidades del nuevo tipo dentro del marco; estas son preguntas internas; $y$ segundo, preguntas sobre la existencia o realidad del sistema de entidades como un todo, llamadas preguntas externas. (Carnap, 1950, 23; énfasis mío)

A pesar de incurrir en cierto anacronismo por no demarcar meridianamente si las nociones de "sistema lingüístico" y "marco lingüístico" son equiparables, Friedman entiende su propuesta como una actualización de la descripción carnapiana de las teorías físicas como marcos lingüísticos estratificados en dos dimensiones. Según Friedman $(2001,45-46)$ una teoría está dividida en dos niveles: el de los enunciados empíricos, enfrentados a la experiencia a través de la experimentación, y el de los principios constitutivos a priori, compuestos éstos por las leyes fundamentales de la teoría y los principios fundamentales de la geometría matemática empleada en el cálculo. Estos dos niveles, junto con un tercero en el

\footnotetext{
${ }^{5}$ Véase Friedman $(1999,169)$ a este respecto.
} 
que me detendré en el apartado 2.3, constituyen la organización conceptual de las teorías físicas.

La distinción entre principios constitutivos y enunciados empíricos presenta al menos tres posibles fuentes de confusión. En primer lugar, no ha de comprenderse la distinción análoga a aquella entre enunciados teóricos y empíricos. Cualquier enunciado empírico aceptado en una teoría es susceptible de ser elevado al rango de principio constitutivo en otra posterior. Einstein, según la lectura de Friedman, tomó los resultados empíricos del experimento del interferómetro de Michelson-Morley, que en la mecánica newtoniana eran interpretados como enunciados empíricos, y resultaron ser elevados por Einstein al estatus de un principio constitutivo en la relatividad especial bajo la formulación del principio de constancia de la velocidad de la luz (Friedman, 2001, 86). La distinción entre principios constitutivos y enunciados empíricos no viene dada por los términos que aparecen en ellos, ni tampoco por su "relativa resistencia a la revisión" empírica (Friedman 2002, 180). En segundo lugar, tampoco debe errarse al comparar ingenuamente los enunciados empíricos con las reglas-P, y los principios constitutivos con las reglas-L. La conclusión es que no existe una definición axiomática de los principios constitutivos; lo que permite demarcar la división sucintamente es si el enunciado en cuestión juega un rol constitutivo en la teoría según el sentido visto en el apartado 1., y esto tan solo es determinable a través del análisis conceptual teniendo como base la historia de la ciencia. Es en este último sentido en que debe establecerse la comparación entre Friedman y Carnap.

Tampoco debe confundirse la estructura conceptual de una teoría con los niveles de organización de la ciencia ${ }^{6}$. Mientras que la primera idea hace referencia a la posición que ocupan las leyes, conceptos, modelos y similares en el ordenamiento conceptual de una teoría (siendo aquí donde debe localizarse la distinción entre principios constitutivos y enunciados empíricos), la segunda estudia la relación entre distintas disciplinas científicas en términos de unificación o pluralismo. La distinción es pertinente ya que el problema de la unidad de la ciencia marca un perspicuo desencuentro entre Carnap y Friedman: mientras que el proyecto intelectual del primero consiste en reducir las cuestiones filosóficas a una Wissenschaftslogik como proceso de purificación científica de la filosofía (Carnap, 1934, \$72), Friedman reivindica que "la filosofía no necesita, y de hecho no debe, perseguir un estatus científico" $(2001,44)$. No es mi intención profundizar en el debate, por lo que asumiré, en pos de los propósitos del artículo, que la organización conceptual de una teoría puede ser investigada sin entrar en a valorar la relación que dicha teoría mantiene con otras disciplinas. De todos modos, más que la relación entre los niveles de una teoría, a Friedman le preocupaban los problemas dinámicos derivados del cambio teórico: a Friedman le preocupaba la inconmensurabilidad.

${ }^{6}$ Véase Potochnik $(2017,163-170)$ para una discusión reciente. 


\subsection{Kuhn, Friedman y la concepción historicista de la ciencia}

Hay dos aspectos de la filosofía historicista de Kuhn con los que Friedman muestra especial sintonía. El primero es la tesis por la que las revoluciones científicas poseen una estructura identificable que distingue entre estadios estables de resolución de rompecabezas y periodos en los que las bases conceptuales de la de la disciplina en cuestión son reformuladas (Kuhn, 1962). En estos segundos periodos, entran en juego lo que Friedman acuñó como meta-paradigmas filosóficos, un entramado permanente de conceptos filosóficos cuya función es servir de orientación al conocimiento científico, proporcionar "nuevas ideas", "programas alternativos" y "posibilidades extendidas" (Friedman, 2001, 17), así como "motivar y sostener la transición de un paradigma o esquema conceptual a otro" (Friedman, 2001, 44). A su juicio, los científicos exploran los recursos intelectuales propios del discurso filosófico cuando los principios constitutivos se ven amenazados durante periodos de crisis científicas. Así Newton edificó su noción de espacio absoluto como respuesta a la metafísica cartesiana (Friedman, 2001, 44) y Einstein aplicó las intuiciones convencionalistas de Poincaré a la concepción del tiempo y al principio de simultaneidad (Friedman, 2001, 23). Como se hará ver más adelante, la existencia de meta-paradigmas será la punta de lanza de las más mordientes críticas a Friedman.

El segundo aspecto compartido es que, para ambos, una ciencia puede considerarse madura cuando comparte un núcleo de presupuestos bien asentados que no son cuestionados por la comunidad. Friedman considera, de hecho, que cuando Kuhn y él señalan los elementos constitutivos de las teorías, están señalando una y la misma cosa: "los principios relativizados a priori", mantiene Friedman, "constituyen lo que Kuhn denomina paradigmas: un conjunto al menos relativamente estable de reglas del juego" cuya misión es "definir o hacer posible las actividades de resolución de problemas" (Friedman, 2001, 45). Aquí es necesario hacer dos precisiones. En primer lugar, Kuhn sugiere que "la existencia de un paradigma no requiere que exista ningún conjunto de reglas" (Kuhn, 1962, 44) porque gran parte del conocimiento que maneja el científico tiene la forma de conocimiento tácito, esto es, conocimiento no explicitado en reglas y principios. Sin embargo, en la concepción de Friedman, los elementos constitutivos a priori son las leyes fundamentales y los sistemas de geometría utilizados en el cálculo, y estos son elementos a todas luces explícitos. El conocimiento tácito al que apela Kuhn incluye una lista de factores constitutivos de un paradigma mucho más amplia que lo que incluyen los elementos constitutivos de Friedman, entre los que se encuentran diferentes prácticas, métodos, instrumentos y valores. Este hecho hace que la asimilación entre un paradigma y un conjunto de principios constitutivos a priori no pueda ser tomada tan a la ligera. Tampoco creo, igualmente, que la versión más depurada de paradigma, los léxicos kuhnianos (Kuhn 1991, 92-94) y los principios constitutivos puedan ser vistos como análogos. Los léxicos son redes de conceptos semánticamente interdependientes y no estrictamente leyes o principios, aunque puedan de hecho adquirir su significado 
a través de una ley. En cualquier caso, lo que sí comparten "léxicos" y "principios fundamentales" en las propuestas respectivas de ambos autores es su fuerte carácter constitutivo ${ }^{7}$.

\subsection{Racionalidad instrumental y racionalidad comunicativa}

A pesar de los puntos comunes, los caminos de ambos se separan tan pronto como aparece el problema de la inconmensurabilidad. Para Kuhn este fue el más interesante quebradero de cabeza que originó su filosofía (Kuhn, 1993, 228). Para Friedman, por contraposición, la inconmensurabilidad supone una amenaza para la racionalidad de la ciencia y un argumento a favor del relativismo conceptual. A su juicio, "en la medida en que no parece haber acuerdo generalizado sobre qué principios constitutivos gobiernan la transición” de una teoría a otra, "no queda ningún sentido en que dicha transición pueda seguir siendo vista como racional y basada en buenas razones" (Friedman, 2002, 182). Si bien Friedman reconoce que la estructura de las revoluciones científicas debe ser de corte rupturista, no admite las consecuencias negativas para la comunicación interteórica típicamente asociadas a tal posición. En base a ello, Friedman desarrolla su propia caracterización dinámica de las teorías científicas que cito in extenso:

En la medida en que las revoluciones conceptuales profundas o cambios de paradigma son un hecho de la vida científica [...] podemos imaginar que nuestros principios constitutivos actuales representan un estadio de un proceso convergente, por así decirlo, en el que pudieran ser vistos como aproximaciones a principios constitutivos más generales y adecuados que serán articulados en un estadio subsiguiente. Podemos así contemplar nuestra comunidad científica actual [...] como una aproximación a una comunidad de investigación final e ideal que ha alcanzado una racionalidad comunicativa universal y transhistórica sobre la base de principios constitutivos plenamente generales y adecuados, obtenidos en el límite ideal del progreso científico. De hecho, debemos contemplar nuestra comunidad

\footnotetext{
${ }^{7}$ De aquí podemos deducir cuál es la posición de Friedman y Kuhn frente a la posibilidad de obtener un conocimiento sintético a priori, que de hecho se encuentra cercana a la de Reichenbach. Si bien se distancian de la concepción trascendental, universal y apodíctica del conocimiento a priori, los tres entienden que hay determinados elementos en un sistema de creencias (en particular, en las teorías científicas) que gozan del carácter constitutivo de los juicios sintéticos a priori. Reichenbach sostiene que los axiomas coordinativos, que recordemos retienen el segundo sentido aducido de aprioricidad y abandonan el primero, son "equivalentes a los juicios sintéticos a priori de Kant" $(1920,47)$ de la manera en que se ha indicado; Friedman sigue a pies juntillas esta distinción; y Kuhn, a colación de la terminología de Reichenbach, asegura que "mis léxicos estructurados se asemejan al segundo sentido, al relativizado" (1993, 245). El distanciamiento de la cuestión de lo sintético a priori es abiertamente menor en estos tres autores que en los empiristas lógicos. Las obras tempranas de Reichenbach y las de Friedman se posicionan explícitamente en la senda del neokantismo; en cuanto a Kuhn, no fue hasta la última etapa de su trayectoria en que se reconoció como un "kantiano de categorías cambiantes" (Kuhn, 1997, 264).
} 
científica como una aproximación a tal comunidad ideal. (Friedman 2001, 6465; énfasis original)

En la interpretación de Friedman, la racionalidad científica de Kuhn se reduce a la actividad de resolución de rompecabezas durante periodos de ciencia normal (Kuhn, 1962, 35-37). A esta racionalidad la denomina racionalidad instrumental, y refiere a nuestra "capacidad de desarrollar un razonamiento efectivo sobre los medios y los fines encaminado a maximizar nuestras probabilidades de tener éxito en la consecución de un objetivo preestablecido" (Friedman, 2001, 54). Friedman no niega la existencia de estos mecanismos en la práctica científica. El problema es que así tan solo se describe una pequeña parte de la naturaleza de la investigación que nada nos dice sobre cómo tal habilidad es transmitida a través comunidades diferentes. A este problema se añade el hecho de que la racionalidad instrumental no nos permite demarcar las ciencias de las pseudociencias. Mostrar competencia para alcanzar un logro preestablecido con efectividad es algo que pueden satisfacer químicos del estado sólido, químicos partidarios del flogisto y alquimistas. Kuhn intentó capear el temporal enumerando una lista bien conocida de cinco valores epistémicos (precisión, alcance, simplicidad, potencialidad y consistencia) que deben guiar la actividad científica (Kuhn, 1977), y que más adelante definió como "los criterios que los que resuelven los rompecabezas deben sopesar al decidir si un rompecabezas [...] ha sido bien resuelto o no" (Kuhn, 1993, 251). Así Kuhn creía haber mostrado un elemento racional propio de la actividad científica que se mantiene tras toda revolución, y que permitía además discriminar disciplinas pseudocientíficas; aunque siendo honestos, a Kuhn le interesaba bastante lo primero y casi nada lo segundo.

Friedman no cree que la comunicación interteórica venga dada por una serie de reglas epistémicas, sino por lo que denomina racionalidad comunicativa. Esta idea de racionalidad, explícitamente extraída de Habermas (1981), remite a nuestra "capacidad de desarrollar un razonamiento argumentativo entre unos y otros encaminado a propiciar un acuerdo o consenso" (Friedman, 2001, 54). Si la racionalidad instrumental apelaba al consenso que una comunidad sostiene en relación a los problemas pertinentes y a las estrategias preferentes de resolución, la racionalidad comunicativa aspira a que los miembros de secuencias de comunidades científicas desarrollen sus principios constitutivos sobre la base consensuada de lo que Hempel denominó el vocabulario anteriormente disponible. Se trata, en definitiva, de que en el paso del concepto de movimiento en Aristóteles al concepto de movimiento acelerado de la mecánica clásica, o en el paso de tal concepción hasta la descripción de trayectorias geodésicas de la física relativista, los nuevos principios constitutivos originados sobre la caracterización del movimiento muestren una "continuación natural de los antiguos conceptos y principios" de manera que "a pesar de que los integrantes del nuevo marco hablen un lenguaje de hecho inconmensurable", estén racionalmente justificados a "utilizar recursos empíricos y conceptuales ya disponibles” (Friedman, 2001, 101). De 
esta manera, la concepción del cambio teórico de Friedman trata de combinar lo que en la epistemología kantiana son dos ideas bien diferenciadas: el sentido constitutivo de los juicios a priori y el uso regulativo de la razón. Creo pertinente dedicarle unas líneas más a este asunto.

Es sabido que para Kant la facultad de la razón debe avanzar siguiendo una serie de principios regulativos, que podemos traducir aquí como reglas metodológicas que orientan la buena investigación y que "sirven como fundamentos de la perfección de ciertas acciones” (Kant, 1987 A569/B597). Estos principios no son sino ideales operativos que deben encauzar toda actividad epistémica y por tanto son diferentes de los juicios sintéticos a priori: mientras que los primeros son regulativos y son producto la razón, los juicios sintéticos a priori son constitutivos y son producto del entendimiento y la sensibilidad. Contrariamente, Friedman entiende que el dominio de lo constitutivo y lo regulativo se entrelaza: los denominados "principios constitutivos" cumplen además una función regulativa. Esto se debe a que, como hemos visto en el anterior extracto citado, para Friedman los principios constitutivos representan "un estadio de un proceso convergente", y caracterizan una comunidad que podemos entender como "una aproximación a una comunidad de investigación final". El uso regulativo de la razón, para Friedman, está inexpugnablemente vinculado a una concepción convergentista de la ciencia: un principio es regulativo cuando puede ser visto como el último eslabón disponible hacia la comunidad de investigación ideal, y en la medida en que los principios constitutivos satisfacen esta definición, son considerados también regulativos. Como se hará ver en el apartado 3.2., lo cierto es que no está tan claro que los principios constitutivos puedan cumplir a su vez una función regulativa, no al menos en un sentido kantiano del término. A mi juicio, lo más próximo a la fórmula kantiana del uso regulativo de la razón en la concepción de Friedman nos remite a los meta-paradigmas filosóficos. Los meta-paradigmas filosóficos, recuérdese, tienen la función de "mediar en la transmisión de la racionalidad (comunicativa) a través de los cambios revolucionarios de paradigma" de tal manera que "la reflexión sobre el nivel meta-paradigmático nos ayude a definir, durante la transición revolucionaria en cuestión, qué es lo que entendemos por una continuación natural entre principios constitutivos razonable y responsable" (Friedman, 2001, 105; énfasis mío). Esto último sí parece la descripción de una genuina función regulativa; pero como se mostrará en el apartado 3.1., la introducción de los meta-paradigmas filosóficos en el esquema comporta dificultades insoslayables.

\subsection{Racionalidad comunicativa y pragmatismo}

Me interesa ahora introducir la siguiente cuestión: si la concepción de Friedman conserva algunas intuiciones nucleares de las elucidaciones de Carnap y Kuhn, ¿cuáles son las razones que justifican su disconformidad respecto al cambio teórico? Carnap y Kuhn no ofrecen ciertamente una imagen homogénea ni mucho 
menos unificada, aunque se ha defendido en ocasiones que sus propuestas pueden ser compatibles (Tsou, 2015, 54) e incluso complementarias (Irzik y Grünberg, 1995). Aquí se entiende que, salvando las distancias, efectivamente Carnap y Kuhn ofrecen al menos un nodo común central en su lectura de los cambios revolucionarios: la consideración de factores de índole pragmático a la hora de evaluar un cambio de teoría ${ }^{8}$. En reiteradas ocasiones, Carnap (1950) ha defendido que lo que determina la elección de un marco lingüístico son "cuestiones", "problemas" y "decisiones prácticas". Y del mismo modo, Kuhn ha insistido en que "la justificación de los léxicos o del cambio de léxico solo puede ser pragmática" (Kuhn 1993, 244). Esta mirada pragmatista sobre el cambio teórico no es tenida por problemática por ninguno de ambos. Para Carnap, las cuestiones metafísicas sobre el realismo o la correspondencia que puedan derivarse sobre tal posición no son asuntos pertinentes; y respecto a Kuhn, su filosofía siempre ha tratado de lidiar con el asunto de la manera más elegante disponible, originando lo que puede denominarse un agnosticismo metafísico ${ }^{9}$.

El caso es que lo que para Carnap y Kuhn es un elemento indispensable en los procesos de elección teórica, para Friedman hace de las teorías científicas una "caja negra", un mero instrumento "con éxito predictivo" (Friedman, 2001, 85 y 53). Esta crítica que parece sugerir que las filosofías de Carnap y Kuhn recaen en el problema señalado por el conocido argumento del no milagro (Putnam, 1975, 73; van Fraassen, 1980, \$7). Como se ha indicado anteriormente, dudo que Carnap mostrase el menor interés por tal problema (de hecho, todo lo contrario: este es el tipo de problemas filosóficos que pretendía purificar), y considero que la caracterización propiamente kuhniana muestra suficientes rasgos internalistas (en cierto sentido más que la de Friedman) como para inferir que en su lectura

${ }^{8}$ Un revisor anónimo ha destacado que esto no hace de Carnap un pragmatista. Esto es absolutamente cierto, como lo es el hecho de que la filosofía de Carnap se adhiere mejor a un marco convencionalista. Con la anterior afirmación he tratado de poner de manifiesto dos ideas: en primer lugar, que el lugar en que las propuestas de Kuhn y Carnap pueden encontrarse (y quizá sea el único), es en la atención a los elementos pragmáticos del cambio teórico; y, en segundo lugar, que esta actitud es justo lo que distancia a ambos de Friedman. Aun así, la distancia que separa las caracterizaciones de Carnap y Kuhn sobre el cambio de teoría sigue siendo mayúscula. Tal y como veo las cosas, la principal diferencia reside en que, si bien Kuhn estaría de acuerdo en que el filósofo de la ciencia debe introducir en su análisis una caracterización de los elementos pragmáticos del conocimiento científico, Carnap negaría que la Wissenschaftslogik deba preocuparse lo más mínimo por ello aun reconociendo su existencia. Otro foco conflictivo está en el hecho de que, "para mí [Kuhn] el cambio de lenguaje es cognitivamente significativo, mientras que para Carnap no lo era" (Kuhn, 1993, 228), por no hablar de que la concepción carnapiana del cambio pragmático se limita a los marcos lingüísticos, a la elección convencional sobre lenguajes lógicos artificiales, mientras que el cambio de lenguaje para Kuhn es tan solo una fracción del cambio teórico, el cual incluye además la sustitución de lo que denominó el "conjunto de compromisos" de la comunidad (Kuhn, 1962, 6). Véase Tsou (2015) para una revisión reciente de los encuentros y desencuentros.

${ }^{9}$ Véase Kuhn $(1993,243)$ para una síntesis de su postura. 
la ciencia es un mero "instrumento con éxito predictivo" ${ }^{10}$. La posición que sostengo, en línea con la de Richardson $(2010,283)$, es que el total descontento de Friedman con una respuesta pragmatista al problema del relativismo le llevó a buscar argumentos de otra índole en nuevos jardines. De ser exitosa su novedosa dinámica de la razón, entonces las indicaciones de Carnap y Kuhn deberían ser abandonadas. Pero, como trataré de mostrar en el siguiente apartado, la propuesta de Friedman aquí descrita dista de ser inmaculada.

\section{Tres argumentos contra Friedman}

En lo que sigue desarrollaré tres argumentos recientes para mostrar que la solución de Friedman al cambio teórico recae en severas dificultades que amenazan la viabilidad de su proyecto filosófico. El primero de ellos denuncia la falta de justificación epistémica de los principios constitutivos (Massimi, 2005; Shaffer, 2011). El segundo cuestiona que la noción de racionalidad comunicativa realmente solucione el problema de la inconmensurabilidad (Richardson, 2002; Van Dyck, 2009; Korkut, 2011). El tercero se centra en la plausibilidad de aplicar el modelo dinámico de Friedman a la historia de la ciencia (Everett, 2015). Esto me dará pie a razonar que a partir de una lectura pragmatista del a priori relativo uno está en condiciones de aportar una descripción más plausible de las teorías científicas.

\subsection{Falta de justificación epistémica de los principios constitutivos}

Como se ha visto en el apartado 2.3, la noción de "continuación natural" que emplea Friedman para explicar el surgimiento racional de nuevos principios puede ser interpretada como un proceso de "elevación epistémica" por la que algunos enunciados empíricos anteriormente disponibles pasan a desempeñar el rol de principios constitutivos en la sucesora. De ahí se infiere que un asunto importante en la constitución de nuevas teorías es la decisión tomada por los científicos sobre qué enunciados empíricos elevar. Esto es algo problemático como bien ha apuntado Massimi (2005). Al proceso de "elevación epistémica" asentada en una decisión tomada por los agentes, sostiene Massimi, le acompaña otro de "eliminación", esto es, una decisión sobre a qué principios constitutivos se les desposee de su anterior carácter constitutivo. Este es un punto que parece ignorar Friedman. Pues si bien es asumible que los resultados del experimento de Michelson-Morley fueron elevados al estatus de un principio constitutivo por Einstein a través del principio de constancia de la luz, también en la relativi-

\footnotetext{
${ }^{10}$ Recuérdese que para Kuhn lo que guía la elección de teoría son valores genuinamente epistémicos. Que la filosofía de la ciencia deba incorporar en sus análisis valiosas enseńanzas de la sociología y la psicología social para comprender las comunidades que acometen tales cambios es algo totalmente distinto.
} 
dad general se sustrae todo carácter constitutivo a las leyes del movimiento de Newton, pasando estas a ser vistas como un caso-límite de la teoría, adecuadas para establecer cálculos en eventos macroscópicos a velocidades sustancialmente inferiores a la velocidad de la luz. Lo que sucede, como sostiene Massimi, es que "en última instancia, la evidencia empírica no puede ser veredicto de un principio constitutivo, ni decidir su fortuna", pues en vista de la importancia nuclear del componente decisivo del agente, "una vez el nuevo marco con sus principios constitutivos ha sido presentado, la fuerza nomológica y la acreditación de tales principios se antoja independiente de la evidencia empírica" (Massimi, 2005, 19). Desde esta perspectiva, Friedman parece dejar la justificación epistémica de los principios constitutivos (y del cambio teórico) no solo en "la genialidad de un solo científico" (Massimi, 2005, 20), sino en las razones subjetivas de los agentes que tanto se esforzó en evitar. Así, su teoría reclamaría, más que un estudio histórico de la transición de la mecánica newtoniana a la relativista, uno psicológico acerca de las razones que accionaron el genio de Einstein.

La crítica de Massimi es meritoria, pero no definitiva. Aquí habría que admitir, a favor de Friedman, que la existencia de creatividad en la actividad científica no menoscaba su racionalidad, ni mucho menos hace de ella una empresa arbitraria. Al contrario, la formulación de propuestas originales bien fundadas supone uno de los motores básicos de la evolución científica. De todos modos, recientemente Shaffer (2011) ha propuesto un perentorio nuevo argumento contra la justificación epistémica de los principios constitutivos que no se dirige al elemento subjetivo del cambio teórico, sino a la noción de meta-paradigma filosófico. Si, cuando una teoría cualquiera entra en crisis, lo que incentiva la proliferación de nuevos principios constitutivos son los recursos conceptuales proporcionados por la filosofía, entonces parte de la justificación epistémica de los principios constitutivos recae sobre la justificación epistémica de los meta-paradigmas filosóficos. Partiendo de este razonamiento, Shaffer concluye que "si no hay condiciones de racionalidad sobre los principios a partir de los cuales son revisados los principios constitutivos", esto es, sobre los meta-paradigmas filosóficos, entonces "no existen motivos convincentes para adoptar un conjunto de principios constitutivos en lugar de otros" (Shaffer, 2011, 203). Efectivamente, Friedman no formula una caracterización normativa sobre qué son los marcos filosóficos, como tampoco se detiene a caracterizar posibles tipos de estrategias plausibles para su correcta utilización. Su dinámica goza así de una envidiable precisión a la hora de comparar las transiciones de una teoría científica a otra en el plano conceptual, pero deja en el aire los mecanismos de transferencia e imbricación entre la filosofía y la ciencia. El problema del convencionalismo y del pragmatismo, para Friedman, era la ausencia de motivos racionales para la adopción de una u otra teoría; lo que muestra Shaffer es que el recurso friedmaniano a los meta-paradigmas no solventa las dificultades que advertía en las corrientes que pretendía evitar. 


\subsection{Sobre el concepto de racionalidad comunicativa y el uso regulativo de la razón}

De la apuesta por una racionalidad comunicativa, universal y transhistórica ejercida través del uso regulativo de los meta-paradigmas filosóficos, Friedman infiere una imagen acumulativista, convergente y progresiva de la ciencia (Friedman, 2001, 65) ${ }^{11}$. Esta caracterización ha sido el foco de numerosas críticas, tanto por las consecuencias de tales posturas, como por la manera de justificarlas. Un primer inconveniente que plantea Richardson (2002) es que no está claro qué quiere expresar Friedman con la idea de un "consenso transhistórico". La racionalidad comunicativa, para Habermas (1981), implica un proceso de deliberación argumentativa que cumple, al menos, dos requisitos: 1) que el diálogo no sea coercitivo, sino consensuado, y 2) que los participantes superen sus puntos de vista inicialmente subjetivos. ¿Cómo pueden un newtoniano y un einsteiniano mantener un diálogo que se asemeje lo más mínimo a lo que propone Habermas? ¿Qué tipo de consenso se espera establecer? Aquí entiendo, con Richardson $(2002,263)$, que este punto ha de serle concedido a Kuhn (1962), quien defendió la idea de que las nuevas teorías reescriben su propia tradición disciplinar a la luz de los nuevos problemas, métodos, creencias, y, en definitiva, en virtud su propio punto de vista. Esta no deja de ser una actitud coercitiva que mantienen las nuevas comunidades científicas respecto a las anteriores, por lo que no veo en qué sentido puede establecerse un diálogo consensuado en el que newtonianos y relativistas superen sus iniciales puntos de vista sobre la base de los principios constitutivos de estos últimos. Al contrario, resulta más bien que los relativistas establecen la racionalidad de sus predecesores desde su propio lenguaje.

Friedman no solo se muestra incapaz de solventar el problema de la comunicación interteórica, sino que su lectura del uso regulativo de la razón parece alejarse de Kant, aproximándose más bien a una interpretación hegeliana de la historia en la que se produce un despliegue progresivo y convergente de la razón, ejemplificada en el conocimiento científico, desde los estadios más arcanos hasta una comunidad científica ideal (Richardson, 2002, 262). La objeción más clara en esta línea plantearía que el hecho de asumir la existencia de ciertos principios regulativos no tiene porqué ser acompañado de una lectura convergente o progresiva de la ciencia. Así lo ha interpretado Van Dyck (2009), quien ha defendido que inferir una lectura convergentista de la ciencia a partir del uso regulativo de la razón introduce un elemento de ambigüedad crítico en la teoría de Friedman. "El problema básico", mantiene Van Dyck, es que el ideal regulativo "juega un rol en la articulación de lo que es en definitiva un espacio empírico de razones" (esto es, tiene la función de marcar los parámetros de las buenas y las malas ra-

\footnotetext{
${ }^{11}$ Por acumulativista, se entiende que las nuevas teorías incorporan alguna parte del contenido epistémico no rechazado de sus predecesoras; por convergente, que la ciencia está encaminada hacia la consecución de una teoría unificada; y por progresiva, que las teorías sucesoras son en algún sentido mejores o preferibles que sus predecesoras
} 
zones), y en tal contexto "no está claro qué sentido tiene hablar de convergencia" (2009, 698). Recuérdese cuál fue siempre el objetivo de Friedman: otorgar una noción de racionalidad trans-paradigmática como complemento a la teoría de la racionalidad de Kuhn. Pero vistas las cosas, el verdadero problema de la lectura convergentista y acumulativista que propone Friedman emerge justamente al congeniarla con una interpretación kuhniana de las revoluciones científicas ${ }^{12}$.

\subsection{Aplicación del modelo dinámico a la historia de la ciencia}

La interpretación friedmaniana de las revoluciones científicas funciona aceptablemente bien respecto a la revolución física de principios de siglo $\mathrm{XX}$, pero su aplicación dista de ser intuitiva en ciencias cuya fundamentación matemática es menor (Richardson, 2010, 286). A pesar de ello, Friedman ha especulado en varias ocasiones $(2001,120-129 ; 2011)$ con la aplicabilidad de su modelo a otras ciencias especiales sin un resultado meridiano. La fuerza de su modelo se basa en la aplicabilidad a la revolución física de principios del siglo XX. En contra de esta presunta adecuación, Everett (2015) ha examinado ciertos problemas históricos en la lectura de Friedman (2010). Friedman otorga un "lugar central al ejemplo de Einstein de un sistema de referencia en rotación uniforme" porque fue el uso que hizo Einstein del "sistema de referencia en rotación, particularmente, lo que hizo posible en primer lugar una geometría tetra-dimensional" (Friedman, 2010, 725). Si los resultados del experimento de Michelson-Morley dieron lugar al establecimiento del principio de constancia de la luz como principio constitutivo de la relatividad especial, el experimento mental de los sistemas de referencia en rotación dio lugar a la formulación del principio de equivalencia constitutivo de la relatividad general. Esta lectura establece un orden cronológico entre los eventos que justifica el paso de enunciados empíricos a principios constitutivos. Si no fuera cierto que Einstein se enfrentó primero al experimento mental y posteriormente extrajo de él un principio novedoso, entonces el modelo de Friedman no parece adecuarse a los hechos. Esto es precisamente lo que ha tratado de mostrar Everett (2015), sosteniendo que es debatible si antes tuvo lugar la deducción del principio de equivalencia o la consideración del espacio tetra-dimensional como posibilidad física. No pretendo dilucidar aquí quién debería llevarse el gato al agua, pero el hecho de que existan controversias abiertas sobre la adecuación histórica del análisis de Friedman me permite inferir que la aplicación de su modelo dinámico a la historia de la ciencia, incluso en aquellos casos en que parece tener más argumentos a su favor, es problemática. Las tres líneas de crítica analizadas implican que deba buscarse una interpretación alternativa del a priori relativo.

\footnotetext{
${ }^{12}$ Como ha mostrado Korkut (2011), adecuadamente interpretada, la dinámica de la razón "no solo lleva al problema de la inconmensurabilidad" sino que realmente "proporciona sus cimientos filosóficos". Este no tiene porqué ser un resultado negativo, pero sí lo es de cara a las intenciones de Friedman.
} 


\section{4. ¿Hacia una noción pragmatista de la ciencia?}

A lo largo de su obra Friedman manifiesta una clara falta de sensibilidad pragmatista. Esta sensibilidad puede interpretarse de dos formas. En primer lugar, puede entenderse como una desatención deliberada de la bibliografía específica. Hay dos obras capitales sobre el a priori pragmático, las de Lewis $(1923 ; 1929)$ y Pap (1943) que son completamente ignoradas en Dynamics of Reason: tan solo la segunda aparece citada una vez en la cuarta nota a pie, donde se asegura que los pragmatistas "reducen la noción de presuposición al absurdo" (Friedman, 2001, 74; nota 4). No ha sido hasta esta década que la lectura pragmatista ha recibido algo de atención superficial (Friedman, 2012, 49) como respuesta a las sugerencias de Mormann (2012). En su artículo, Mormann argumenta que Friedman "menosprecia los aspectos pragmatistas del conocimiento científico"; concretamente, la manera en que el conocimiento y las prácticas en que se produce están imbricadas, así como las consecuencias que tal imbricación proyecta sobre el concepto de racionalidad. Su análisis de las elucidaciones de Friedman y Kuhn le lleva a concluir que la noción friedmaniana del a priori relativo "está confeccionada a medida de las necesidades de un acercamiento estrictamente teórico del conocimiento científico" $(2012,36)$. Esto es algo que el propio Friedman ha reconocido, identificando humildemente las carencias de una postura "simplificadamente formalista" (Friedman 2010, 697). Partiendo de la lectura de Mormann, parece como si Friedman hubiese asumido que, a pesar de haber identificado la existencia de elementos pragmáticos en el conocimiento científico, estos no debieran preocuparle al filósofo de la ciencia, sino que debieran ser atendidos por algún otro tipo de especialista (véase la nota 12). Lo que me parece indiscutible, de nuevo en sintonía con Mormann, es que una propuesta que se localice en la estela de la filosofía kuhniana necesita considerar en su análisis las dimensiones pragmáticas de la ciencia si aspira a proporcionar una imagen adecuada y real del conocimiento científico. Por ello expondré los acercamientos al a priori relativo desde una tónica pragmatista como alternativa a la concepción de Friedman, tratando de mostrar que existen vías de caracterizar el a priori relativo sin renunciar al elemento pragmático del cambio teórico en las filosofías de Carnap y Kuhn.

Hasta este punto se ha manejado una idea intuitiva de pragmatismo. Cuando se ha afirmado que la elección teórica está permeada por consideraciones pragmáticas, se ha querido sostener que las condiciones que determinan tal decisión dependen de diferentes "valores epistémicos", en palabras de Kuhn, que orientan tal elección. En última instancia, según una perspectiva pragmatista de la elección teórica, lo que la comunidad científica tiene en consideración cuando opta por una nueva teoría son las consecuencias de tal decisión, y no una lista programática de criterios ponderados. Además, se asume que los cambios son progresivos: la comunidad científica no se acostó newtoniana y se despertó relativista. La adopción del nuevo marco teórico fue una transformación escalonada atravesada por las condiciones materiales y la capacidad persuasiva de los nuevos 
científicos (Richardson, 2002). La reciente propuesta de Stump (2015) encaja satisfactoriamente en este amplio contexto. A su juicio, abandonar una serie de principios y adoptar otros es una decisión de base pragmática, condicionada por las necesidades de las comunidades científicas en cuestión. Para Stump (2015, 97), una teoría es una estructura edificada en base a principios funcionalmente a priori cuyo objetivo es simplificar nuestra interpretación de los fenómenos. Esta idea funcionalista fue en primer lugar desarrollada por Pap (1946) en el marco del análisis de las leyes de Newton. La diferencia que presentan la concepción funcional de Pap del a priori y la relativa es que la segunda denomina "rol constitutivo" a lo que la primera se refiere como "rol funcional", pero ambas hacen énfasis en que lo que demarca el estatus epistémico de los enunciados no es su carácter analítico/sintético, sino precisamente el papel que desarrollen en la teoría. Esta es, siguiendo a Stump $(2015,167)$, una lectura deflacionaria de los elementos constitutivos de las teorías "que deja en el centro las creencias sobre los elementos constitutivos" y que aboga por un cambio de índole pragmatista de los mismos. Así interpretada, la estrategia deflacionaria de Stump marida bien con las concepciones de Carnap y Kuhn; y en tanto que Stump no establece criterios de racionalidad trans-teóricos, lo hace mejor que la de Friedman.

Ahora quisiera precisar un sentido más estrecho de "pragmatismo" cercano a la máxima pragmática peirceana. Este remite a lo que Putnam bautizó como la "primacía de la práctica" $(1995,52)$ y puede sintetizarse como el hecho de localizar en el centro de la reflexión las actividades prácticas acometidas por un individuo o una comunidad junto con sus consecuencias. El tipo de actividades deberá precisarse según el campo de estudio. En el caso de la ciencia, las prácticas a analizar son lo que Chang (2012) ha denominado "actividades epistémicas". Según Chang $(2012,16)$, "una actividad epistémica es un conjunto más o menos coherente de operaciones físicas o mentales encaminadas a la producción o la mejora del conocimiento de una forma determinada, en concordancia con algunas reglas discernibles", reglas que por cierto pueden no estar articuladas. Un elemento importante de esta noción de actividad epistémica son los propósitos del agente: "la presencia de un objetivo identificable es lo que distingue las actividades de los meros sucesos físicos de los cuerpos humanos", y concluye sosteniendo que "la coherencia de una actividad se identifica con el grado de éxito en alcanzar su objetivo". Este es el tipo de ejercicio epistémico que corona la ciencia y que supone el día a día de su actividad. Aquí Chang juega un movimiento sugestivo que conecta con el a priori relativo: a su parecer, "si queremos participar en una actividad epistémica concreta, entonces debemos presuponer la veracidad de ciertos principios metafísicos particulares" (Chang, 2008, 113). Si queremos contar, por ejemplo, hemos de suponer que el dominio de objetos contables es discreto; si queremos predecir, hemos de suponer que en el dominio sobre el que recae la predicción se cumple el principio de consecuencia uniforme; si queremos explicar, debemos suponer que el principio de razón suficiente es satisfecho, etc. Este es un tipo de "necesidad pragmática", surgida de "nuestros requerimientos 
de acción epistémica, no de ningún tipo de híper-verdad que pertenece a la proposición" (Chang, 2008, 125). Para Chang, el a priori es relativo en tanto que no hay un principio trascendental que nos impele a incurrir en una actividad epistémica determinada. Y es pragmático en la medida en que involucrarnos en dicha actividad es una elección que reporta beneficios. Ahora bien: sigue siendo a priori en tanto que los principios metafísicos que cita Chang en relación a la actividad en cuestión son necesarios.

La de Chang es una tesis que surge del pragmatismo conceptual de Lewis (1929) y de su proyecto de liberación del a priori. Para Lewis, el conjunto de creencias fundamentales con las que nos comprometemos está atravesada por los fines pragmáticos que devienen de adoptar tal creencia. Esta es una versión del a priori relativo, que Lewis expone de la siguiente manera:

Si fuéramos medusas en un mundo líquido, probablemente no sabríamos sumar en absoluto, porque los beneficios otorgados por tal acto serían ínfimos. Aun así, si una súper-medusa inventase la aritmética [...] no encontraría ninguna experiencia posible a la que contraponerla, y podría aplicarla a sus propias ideas si le reportase beneficio. [...] Los principios geométricos aplicados son verdades a priori de todas las cosas que llenan el espacio. Pero esta verdad a priori tiene su aspecto pragmático en tanto existen alternativas sobre la manera en que la categoría de 'lo espacial' debiera ser acotado. (Lewis, 1929, 252-253)

De nuevo Lewis enfrenta el dilema que inquietó a Kant, Reichenbach y Friedman: la relación entre la experiencia, la naturaleza del espacio y la geometría. Su solución, por el contrario, es bien distinta. Para Lewis, lo que nos lleva a asociar ciertos principios de geometría al espacio físico son sencillamente las consecuencias beneficiosas que este hecho puede acarrear. Estos "beneficios" pueden tener una dimensión epistémica, como la mejoría en la calidad de las predicciones o la simplicidad del nuevo modelo, pero Lewis maneja una idea de beneficio mucho más amplia que impregna lo subjetivo y lo social. Lo que sí parece asumible es que, debido a estos hechos, y a pesar de que la idea de constitución no aparece en la caracterización de Lewis, el a priori relativo y pragmático parece anticipar aspectos importantes del convencionalismo carnapiano y del agnosticismo kuhniano respecto a las consecuencias de la elección teórica ${ }^{13}$. La lectura pragmática es, en definitiva, una vía alternativa para defender la existencia de principios a priori en las teorías científicas sin tener que bregar con las dificultades teóricas que presentan algunos conceptos como "meta-paradigma filosófico", "racionalidad comunicativa" o, en suma, una idea netamente convergentista del conocimiento científico.

\footnotetext{
${ }^{13}$ Recientemente Mayoral (2017) ha destacado los numerosos aspectos comunes entre el a priori pragmático de Lewis y la concepción kuhniana del cambio teórico.
} 


\section{Conclusiones}

El desarrollo del presente artículo ha constado de tres fases. Primero, se ha presentado una lectura panorámica del a priori relativo a partir de su mayor exponente, Michael Friedman, como heredero de las propuestas de Carnap y Kuhn. En segundo lugar, se han atendido las críticas a su modelo. Y, por último, se ha abierto la puerta a la concepción pragmatista del a priori relativo como respuesta a tales dificultades. El objetivo ha sido defender que acoger de manera efectiva las propuestas de Carnap y Kuhn en una intelección adecuada sobre la ciencia pasa por destacar y no esconder las conclusiones que ambos autores establecieron cercanas al pragmatismo. También se ha pretendido hacer ver que esta es la senda que han tomado algunos autores en los últimos años, y que, en vista de ello, las perspectivas auguran un incremento en las nociones pragmatistas de la ciencia.

Friedman acertó en su lectura de Kuhn y Carnap al destacar la importancia del elemento constitutivo que ciertos componentes juegan en una teoría. Pero erró al menospreciar el marcado factor pragmatista de sus propuestas. Concretamente, el asociado al cambio teórico, pero también, respecto a Kuhn, la importancia que poseen lo que Chang ha denominado actividades epistémicas en la caracterización de la naturaleza del conocimiento científico. La imagen de la ciencia que se infiere de la filosofía de Friedman es una enunciativista en la que el lenguaje de la teoría se localiza en el centro de la reflexión. Esto permite llevar a cabo un análisis preciso y pormenorizado de la organización conceptual de la teoría y resulta, de hecho, inestimable en toda caracterización de la idea de constitución. Pero, por otra parte, poner la mira en las relaciones establecidas entre los elementos del lenguaje de la ciencia nos hace desatender aspectos que toda caracterización filosófica de la ciencia debería incluir. Sin ser el único, al que aquí se ha prestado atención es al elemento pragmático de la ciencia. Sería interesante hacer lo propio con las dimensiones axiológicas, materiales o institucionales, entre otras. Este artículo abre así nuevas sendas de investigación localizadas en la pulsión entre las caracterizaciones apriorísticas de las teorías científicas y el análisis de las consecuencias de su dimensión pragmática.

\section{Referencias bibliográficas}

Carnap, Rudolf (1934). Logical Syntax of Language. Abingdon: Routledge [Reedición de 2001].

Carnap, Rudolf (1950). Empiricism, Semantics and Ontology. Revue Internationale de Philosophie, 4(11), 20-40.

Chang, Hason (2008). Contingent Transcendental Arguments for Metaphysical Principles. En Michela Massimi (Ed.), Kant and Philosophy of Science Today (pp. 113133). Cambridge: Cambridge University Press. 


$$
\begin{aligned}
& \text { Mariano Sanjuan Salinas } \\
& \text { El a priori relativo. ¿¿Hacia una noción pragmatista de la ciencia? }
\end{aligned}
$$

Chang, Hasok (2012). Is Water $\mathrm{H}_{2} \mathrm{O}$ ? Dordrecht: Springer.

Coffa, José Alberto (1991). The Semantic tradition: From Kant to Carnap. Cambridge: Cambridge University Press.

De Pierris, Graciela (1992). The Constitutive A Priori. Canadian Journal of Philosophy, 22(1), 179-214.

Everett, Jonathan (2015). The constitutive a priori and the distinction between mathematical and physical possibility. Studies in History and Philosophy of Modern Physics, 52, 139-152.

Friedman, Michael (1994). Kant and the Twentieth Century. En Paolo Parrini (Ed.), Kant and Contemporary Epistemology (pp. 27-46). Dordrecht: Kluwer Academic Publishers.

Friedman, Michael (1999). Reconsidering Logical Positivism. Nueva York: Cambridge University Press.

Friedman, Michael (2001). Dynamics of Reason. Stanford: CSLI Publications.

Friedman, Michael (2002). Kant, Kuhn and the Rationality of Science. Philosophy of Science, 69, 171-190.

Friedman, Michael (2007). Coordination, Constitution and Convention. En Alan Richardson y Thomas Uebel (Eds.), The Cambridge Companion to Logical Empiricism (pp. 91-116). Cambridge: Cambridge University Press.

Friedman, Michael (2008). Einstein, Kant and the A Priori. En Michela Massimi (Ed.), Kant and Philosophy of Science Today (pp. 95-112). Cambridge: Cambridge University Press.

Friedman, Michael (2010). Synthetic History Reconsidered. En Mary Domski y Michael Dickson (Eds.), Discourse on a New Method (pp. 571-801). Illinois: Open Court.

Friedman, Michael (2011). Extending the Dynamics of Reason. Erkenntis 75, 431-444.

Friedman, Michael (2012). Reconsidering the Dynamics of Reason. Studies in History and Philosophy of Science, 43(1), 47-53.

Habermas, Jürgen (1981). Teoría de la acción comunicativa. Madrid: Taurus. [Edición de 1999].

Irzik, Gürol y Grünberg, Teo (1995). Carnap and Kuhn: Arch Enemies or Close Allies? The British Journal for the Philosophy of Science, 46(3), 285-307.

Kant, Immanuel (1787). Crítica de la Razón Pura. México: Fondo de Cultura Económica [Edición de 2009].

Korkut, Buket (2011). The Real Virtue of Friedman's Neo-Kantian Philosophy of Science. Philosophy of Science, 78, 1-15.

Kripke, Saul A. (1980). Naming and necessity. Cambridge: Harvard University Press. 
Kuhn, Thomas (1962). The Structure of Scientific Revolutions. Chicago: The University of Chicago Press [3 Edición de 1996].

Kuhn, Thomas (1977). Objectivity, Value Judgement and Theory Choice. En The Essential Tension (pp. 320-339). Chicago: The University of Chicago Press.

Kuhn, Thomas (1991). The Road Since Structure. En James Conant y John Haugeland (Eds.), The Road Since Structure (pp. 90-104). Chicago: The University of Chicago Press.

Kuhn, Thomas (1993). Afterwords. En James Conant y John Haugeland (Eds.), The Road Since Structure (pp. 224-252). Chicago: The University of Chicago Press.

Kuhn, Thomas (1997). A discussion with Thomas Kuhn. En James Conant y John Haugeland (Eds.), The Road Since Structure (pp. 253-324). Chicago: The University of Chicago Press.

Lewis, Clarence Irving (1923). A Pragmatic Conception of the a Priori. Journal of Philosophy, 20(7), 169-177.

Lewis, Clarence Irving (1929). Mind and World Order. Nueva York: Dover Publications. [Edición de 1990].

Massimi, Michela (2005). Pauli's Exclusion Principle. Cambridge: Cambridge University Press.

Mayoral, Juan Vicente (2017). The Given, The Pragmatic A Priori and Scientific Change. En Peter Olen y Carl Sachs (Eds.), Pragmatism in Transition (pp. 79-102). Estados Unidos: Palgrave.

Mormann, Thomas (2012). A Place for Pragmatism in the Dynamics of Reason? Studies in History and Philosophy of Science, 43(1), 27-37.

Padovani, Flavia (2011). Relativizing the Relativized A Priori: Reichenbach Axioms of Coordination Divided. Synthese, 181, 42-62.

Pap, Arthur (1946). The a Priori in Physical Theory. Nueva York: Russel \& Russel.

Peláez Cedrés, Álvaro (2008). Lo a priori constitutivo: historia y perspectiva. México: Anthropos.

Potochnik, Angela (2017). Idealization and the Aims of Science. Chicago: The University of Chicago Press.

Putnam, Hilary (1975). What is Mathematical Truth. En Mathematics, Matter and Method. Cambridge: Cambridge University Press.

Putnam, Hilary (1995). Pragmatism. Estados Unidos: Blackwell.

Reichenbach, Hans (1920). The Theory of Relativity and A Priori Knowledge. Berkeley y Los Angeles: Cambridge University Press [Edición de 1965].

Richardson, Alan (2002). Narrating the History of Reason Itself. Perspectives on Science, 10(3), 253-274. 
Richardson, Alan (2010). Ernst Cassirer and Michael Friedman: Kantian or Hegelian Dynamics of Reason? En Mary Domski y Michael Dickson (Eds.), Discourse on a New Method (pp. 279-294). Illinois: Open Court.

Ryckman, Thomas (2005). The Reign of Relativity. Oxford: Oxford University Press.

Shaffer, Michael J. (2011). The Constitutive A Priori and Epistemic Justification. En Michael J. Shaffer y Michael L. Veber (Eds.), What place for the A Priori? (pp. 193-210). Chicago: Open Court.

Schlick, Moritz (1925). General Theory of Knowledge. Austria: Springer [Edición de 1974].

Stump, David J. (2015). Conceptual Change and the Philosophy of Science. Nueva York: Routledge.

Tsou, Jonathan Y. (2015). Reconsidering the Carnap-Kuhn Connection. En William J. Devlin y Alisa Bokulich (Eds.), Kuhn's Structure of Scientific Revolutions - 50 years on (pp. 51-70). Dordrecht: Springer.

Van Dyck, Maarten (2009). Dynamics of Reason and the Kantian Project. Philosophy of Science 76, 689-700.

van Fraassen, Bas Cornelis (1980). The Scientific Image. Oxford: Clarendon Press. 